\title{
Pathway to Complete Energy Sector Decarbonization with Available Iridium Resources Using Ultra-Low Loaded Water Electrolyzers
}

Zachary Taie ${ }^{1,2}$, Xiong Peng ${ }^{*}$, Devashish Kulkarni ${ }^{3}$, Iryna Zenyuk ${ }^{3}$, Adam Weber ${ }^{1}$, Christopher Hagen $^{2}$, Nemanja Danilovic ${ }^{1 *}$

${ }^{1}$ Lawrence Berkeley National Laboratory, Energy Technologies Area, Energy Conversion Group, Berkeley, California 94720, USA

${ }^{2}$ Oregon State University, School of Mechanical, Industrial, and Manufacturing Engineering, Bend, OR 97701, U.S.

${ }^{3}$ University of California - Irvine, National Fuel Cell Research Center, Department of Chemical Engineering and Material Science, Irvine, California 92697, USA

*Corresponding authors: xiongp@1bl.gov, ndanilovic@1bl.gov

\section{Supporting Information}

Ir Ink Stability

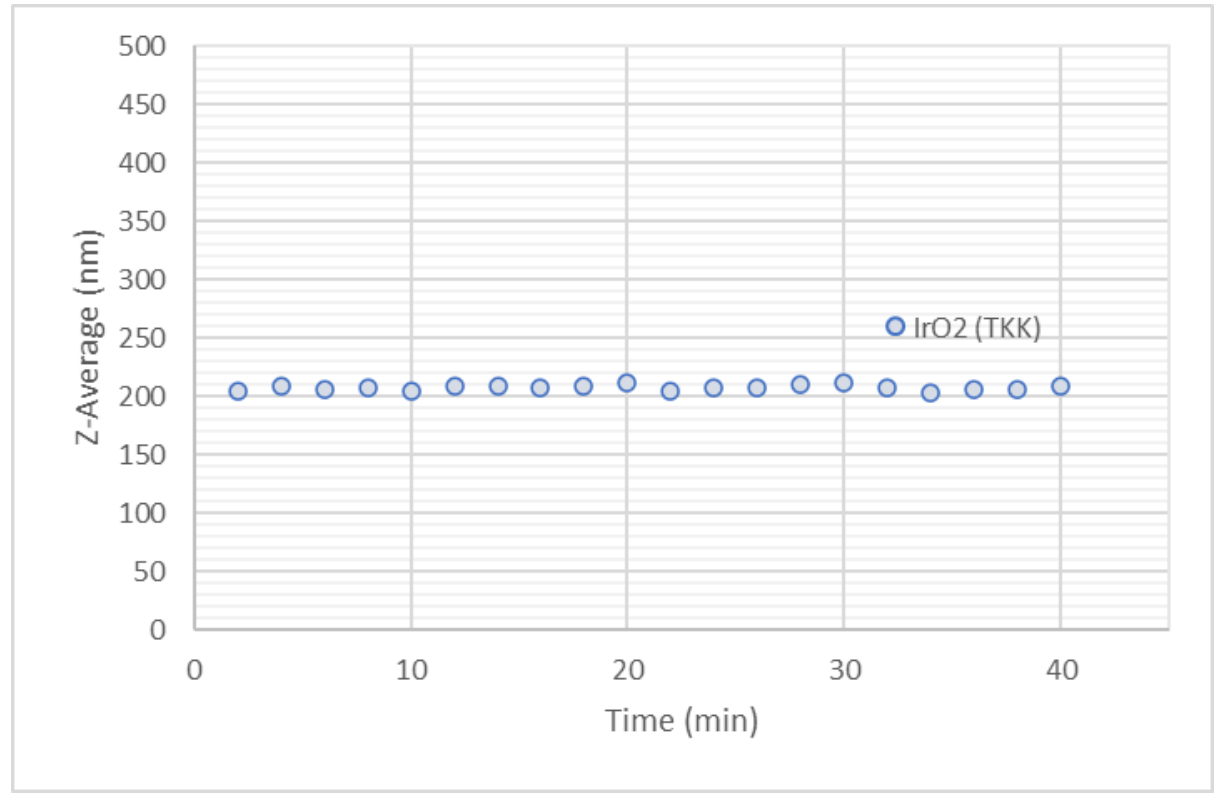

Figure S1. Evolution of average ink agglomerate size vs time. 


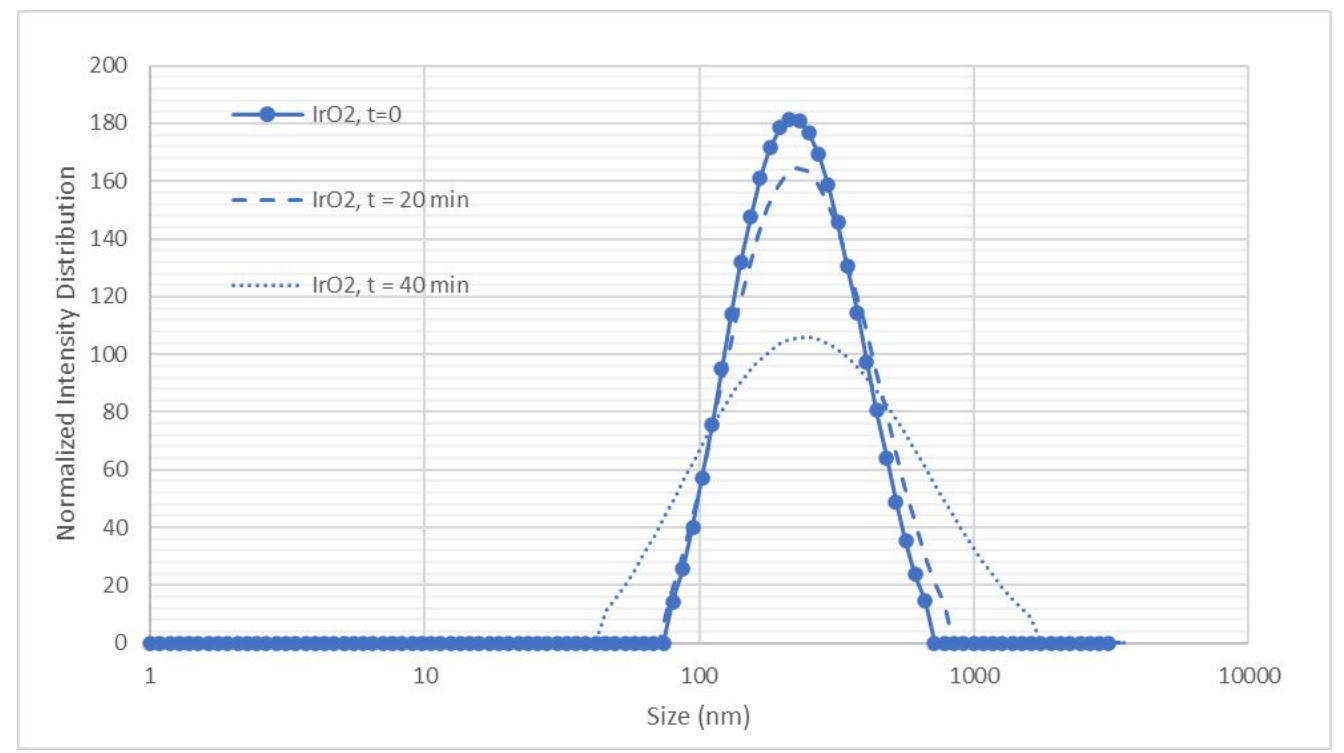

Figure S2. Evolving agglomerate size distribution of Ir ink over 40 minutes.

Figure S1 and Figure S2 show the average Ir ink agglomeration size and distribution as a function of time as measured using dynamic light scattering (DLS). Figure S1 shows the average agglomeration size was nearly unchanged over the 40 minutes of testing which is longer than the longest catalyst coated membrane fabrication time. Figure S2 shows the agglomerate size distribution widens slightly over time, but the mean remains near $200 \mathrm{~nm}$, consistent with Pt/C-Nafion inks which form agglomerates sized between 100-600nm with different water content ${ }^{1}$, or from $\mathrm{IrO}_{\mathrm{x}}$ inks with variable ionomer to catalyst ratios $^{2}$. 


\section{Physical Characterization}

XRF calibration of deposited catalyst layers

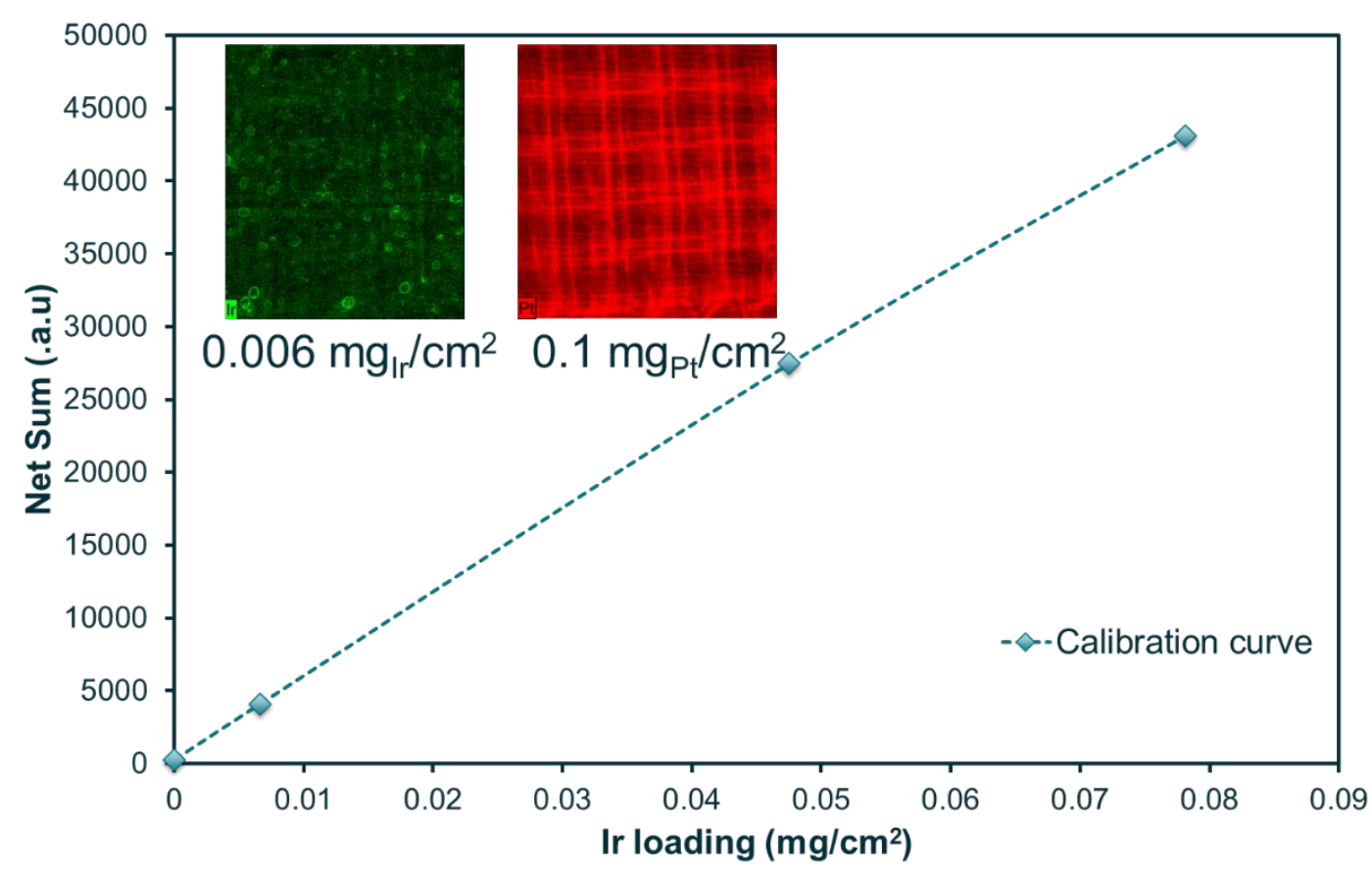

Figure S3. Ir XRF calibration curve.

Three Ir and three Pt standard loadings were purchased from Micromatter Technologies Inc. The standards were scanned (along with a $0 \mathrm{mg} \mathrm{cm}^{-2}$ blank) using a consistent XRF method and the net sum was recorded to produce an intensity vs Ir (or Pt) loading curve. These curves were used to measure the loading of the catalyst coated membranes. 


\section{Micro and X-ray Computed Tomography}

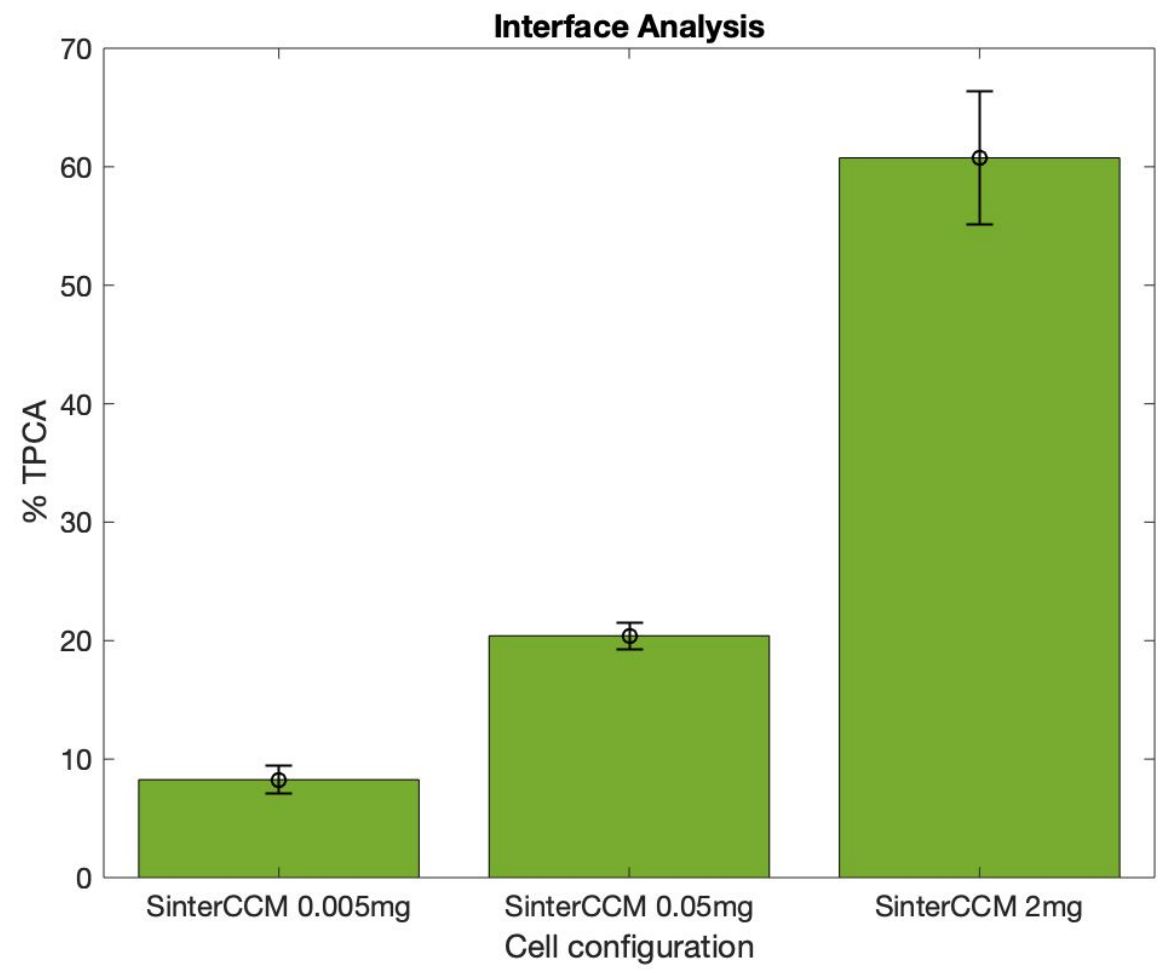

Figure S4. Triple phase contact area (TPCA) percentage.

Percentage of the anode CL-PTL interface where the PTL, CL, and membrane are all in contact. This represents the portion of the CL interface that is likely OER-active since the CL electrical conductivity is believed to limit the activity of sites away from the PTL.

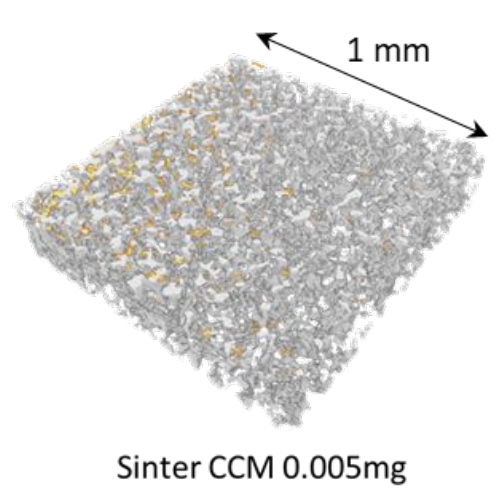

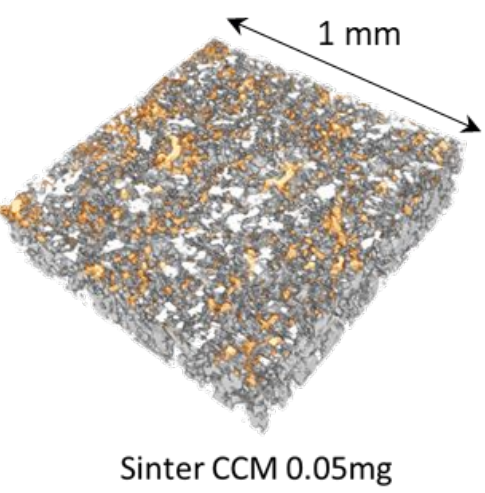

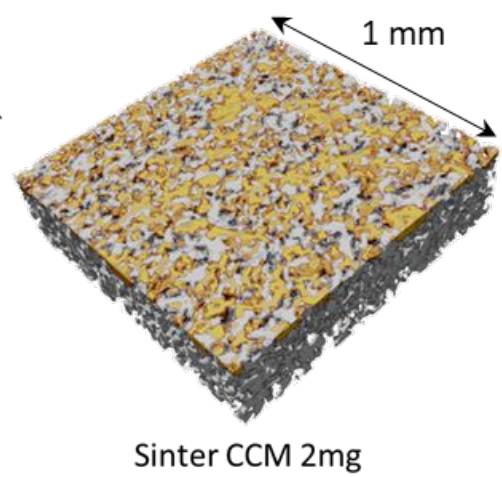

Figure S5. Visual representation of PTL-CL interfacial Ir loading for ultra-low $\left(0.005 \mathrm{mg} \mathrm{cm}^{-2}\right)$, low $(0.05$ $\left.\mathrm{mg} \mathrm{cm}{ }^{-2}\right)$, and commercial $\left(2 \mathrm{mg} \mathrm{cm}^{-2}\right)$ Ir loadings. 
Visual 3-dimensional distribution of Ir in the PTL-CL interface as the loading is decreased. The resolution of the XCT and micro-XCT images are $1.73 \mu$ voxel $^{-1}$ and $0.323 \mu$ voxel $^{-1}$, which is roughly $125-216 \%$ and $25-40 \%$ of the average catalyst-layer thickness, respectively. Therefore, limitations in the signal attenuation and segmentation may result in an incomplete reconstruction of the full CL.
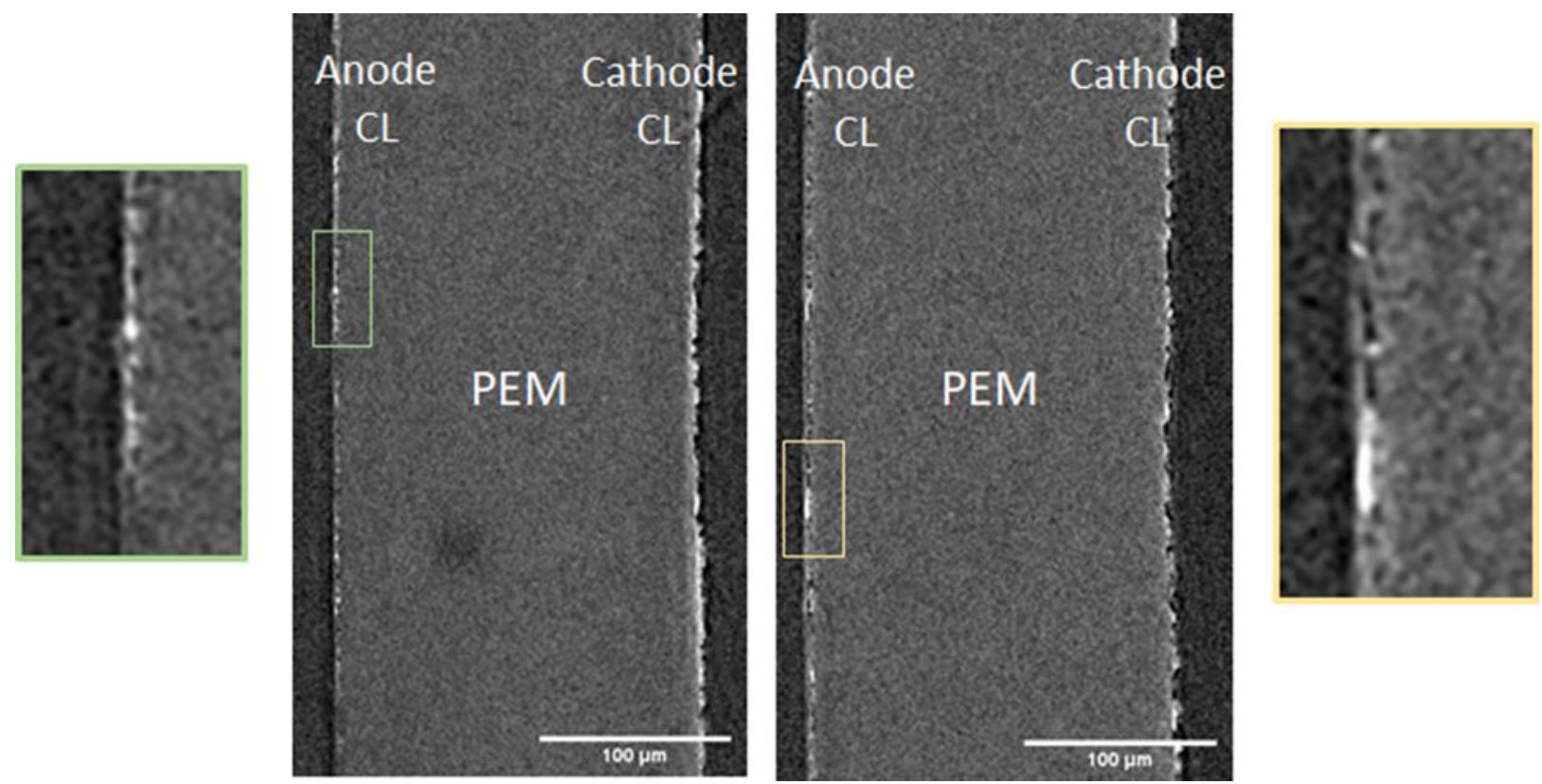

Figure S6. Representative cross section XCT images of $0.05 \mathrm{mg}_{\mathrm{Ir}} \mathrm{cm}^{-2}$ catalyst coated membranes. 

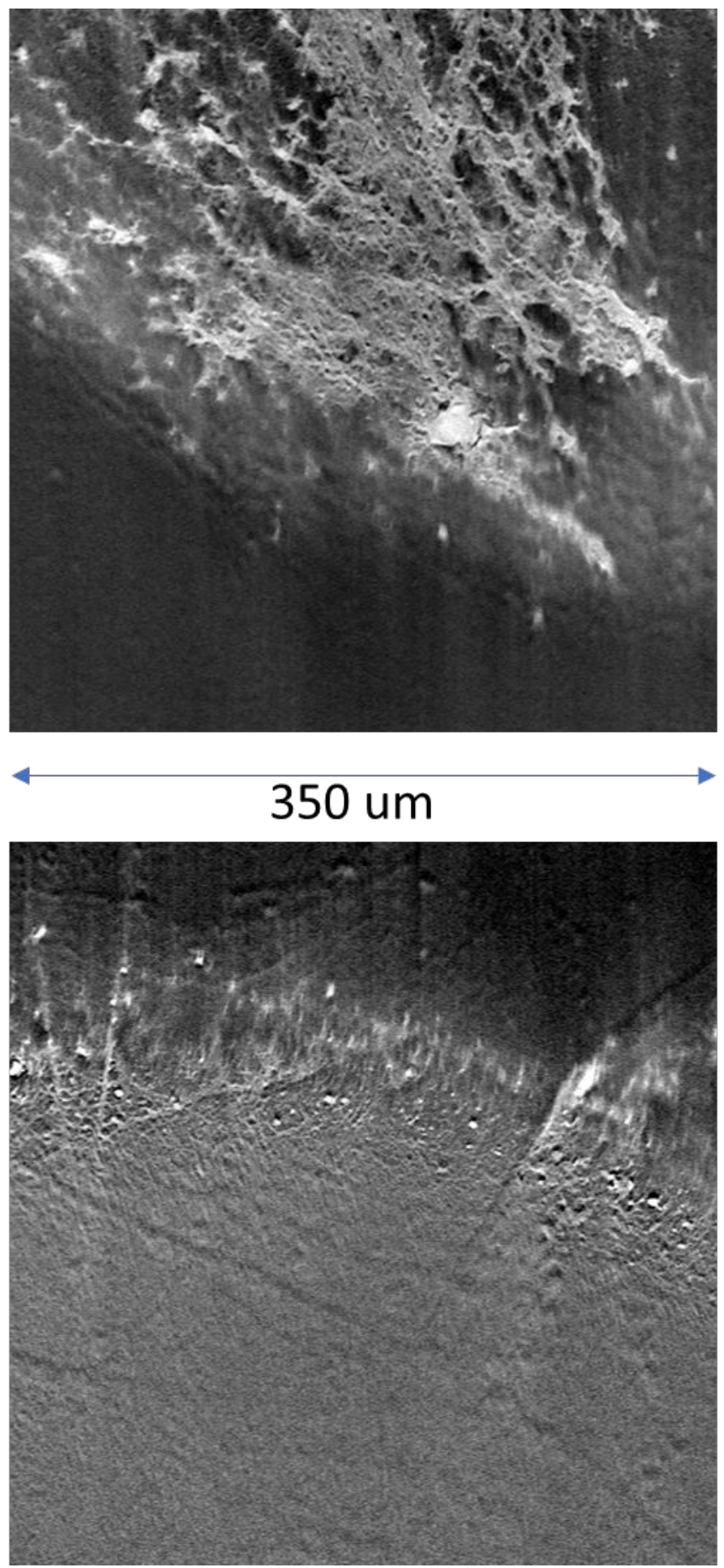

Figure S7. In-plane $\mu$-XCT surface images of the $0.05 \mathrm{mg}_{\mathrm{Ir}} \mathrm{cm}^{-2}$ anode CL. 
Catalyst Layer

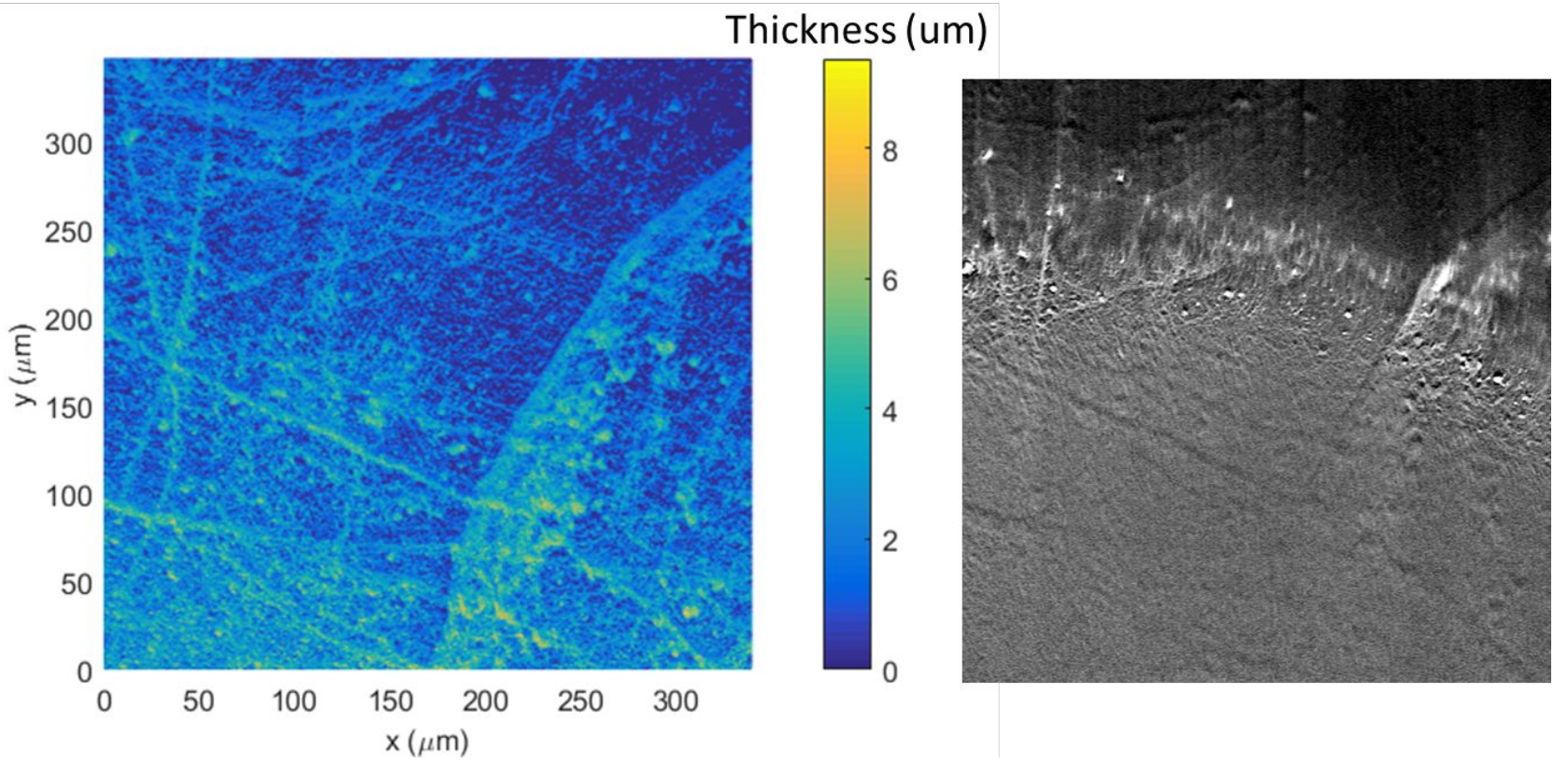

Figure S8. Mapping of anode CL thickness (left) of a representative section of the $0.05 \mathrm{mg}_{\mathrm{Ir}} \mathrm{cm}^{-2}$ loaded catalyst coated membrane.

Figure S6, Figure S7, and Figure S8 show intermediate and alternative $\mu$ - and XCT results of the $0.05 \mathrm{mg}_{\mathrm{Ir}} \mathrm{cm}^{-2}$ loaded catalyst coated membrane. Figure S6 shows representative cross-sectional images of the catalyst coated membrane, showing both the anode and cathode CLs. Figure S7 shows in-plane views of the CL surface that would be in contact with the PTL, and Figure S8 shows a map of CL thickness of one of the representative images. 

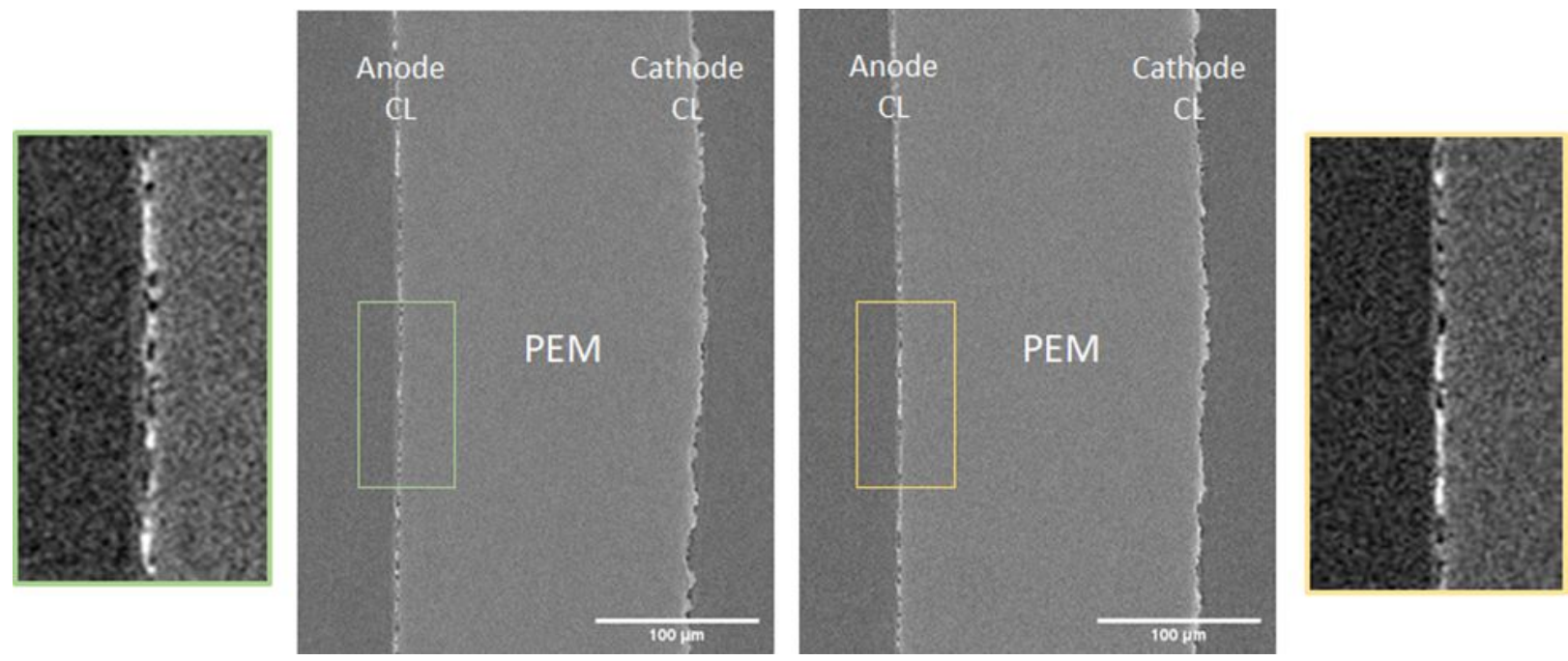

Figure S9. Representative cross section XCT images of $0.005 \mathrm{mg}_{\mathrm{Ir}} \mathrm{cm}^{-2}$ catalyst coated membranes. 

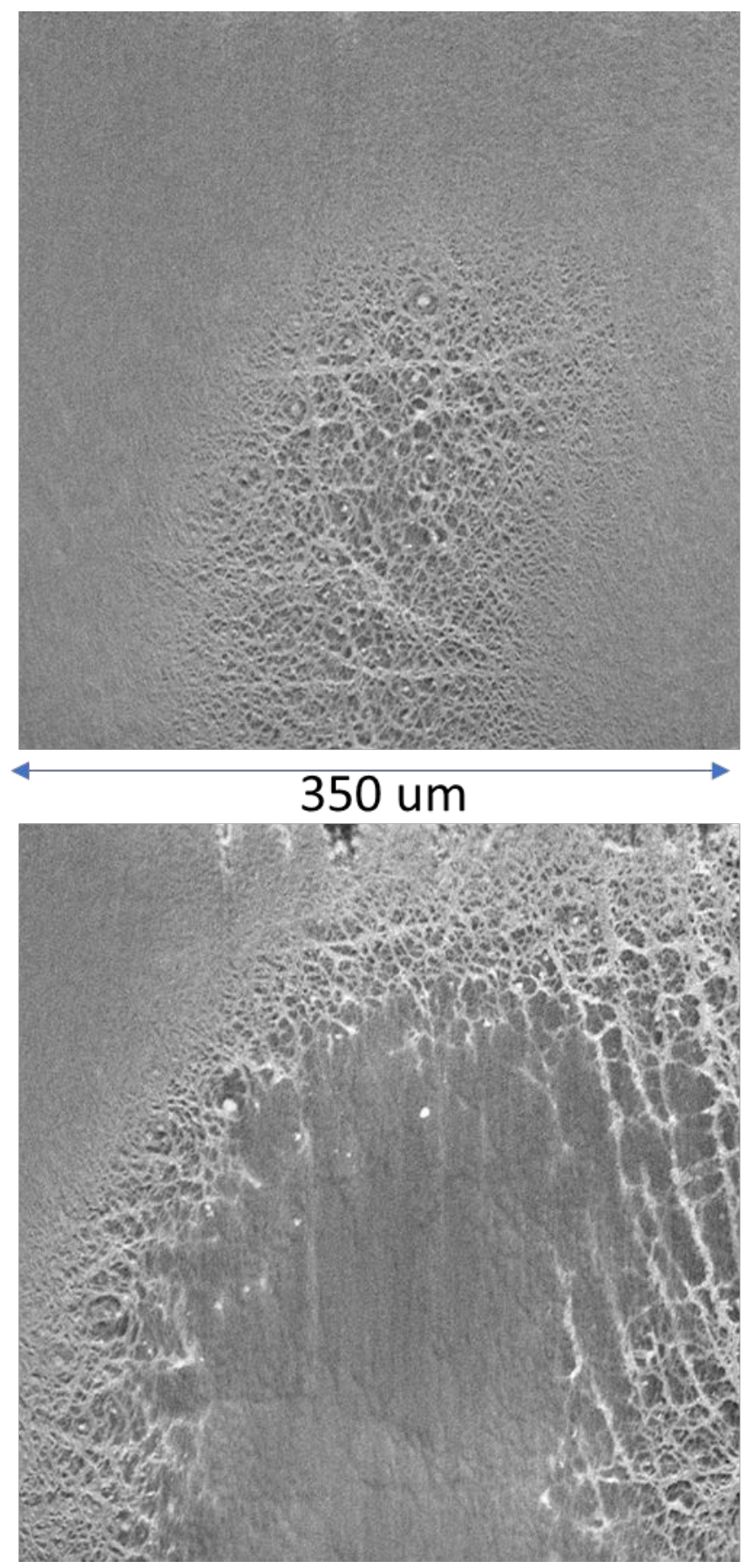

Figure S10. In-plane $\mu$-XCT surface images of the $0.005 \mathrm{mg}_{\mathrm{Ir}} \mathrm{cm}^{-2}$ anode CL. 


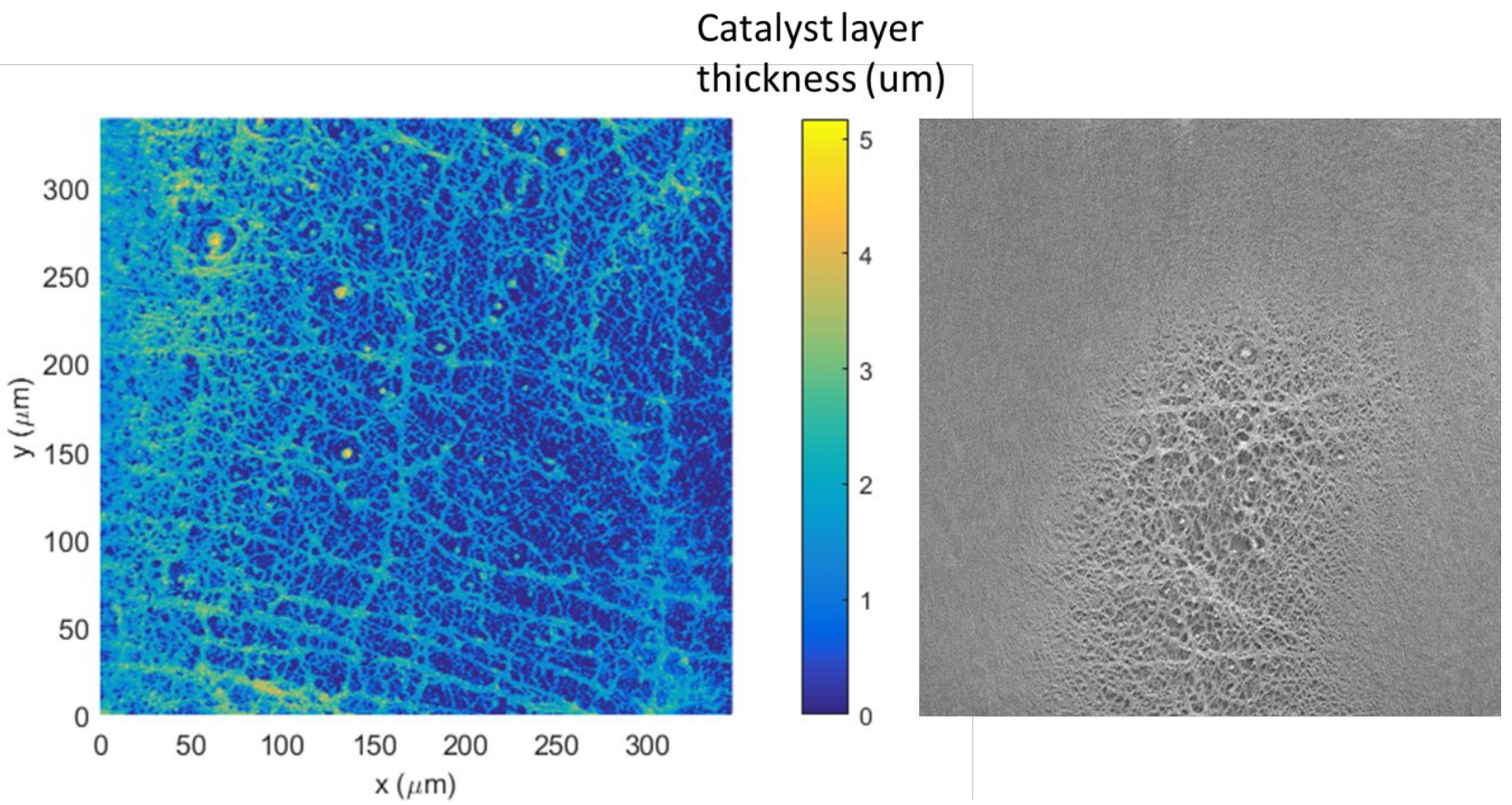

Figure S11. Mapping of anode CL thickness (left) of a representative section of the $0.005 \mathrm{mg}_{\mathrm{Ir}} \mathrm{cm}^{-2}$ loaded catalyst coated membrane.

Like Figure S6, Figure S7, and Figure S8 above, Figure S9, Figure S10, and Figure S11 show intermediate and alternative $\mu$ - and XCT results, though this time of the $0.005 \mathrm{mg}_{\mathrm{Ir}} \mathrm{cm}^{-2}$ loaded catalyst coated membrane. Figure S9 shows representative cross-sectional images of the catalyst coated membrane, showing both the anode and cathode CLs. Figure S10 shows in-plane views of the CL surface that would be in contact with the PTL, and Figure S11 shows a map of CL thickness of one of the representative images. 


\section{Current Density Overpotential Analysis}
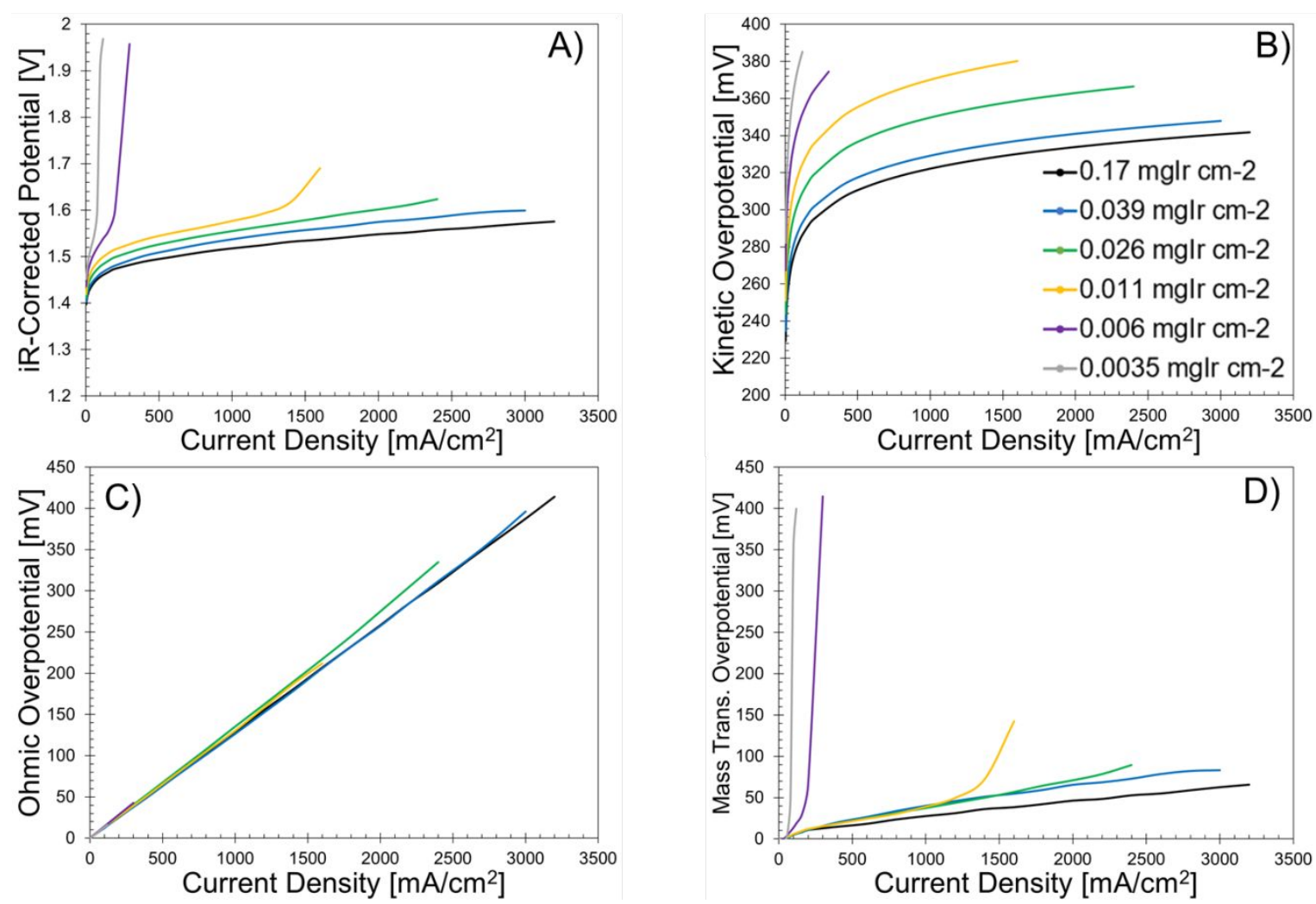

Figure S12. a) PEMWE iR-corrected polarization curves at different Ir loadings under constant ionomer/catalyst ratio of 0.116 ; b) kinetic overpotential, c) ohmic overpotential and d) mass transport overpotential extracted from data shown in Figure 1 and a). PEMWEs were tested under ambient pressure at $80{ }^{\circ} \mathrm{C}$. Cathode Pt loadings were kept at $0.2 \pm 0.02 \mathrm{mg} / \mathrm{cm}^{2}$ for all tests. Nafion ${ }^{\mathrm{TM}} 117$ was used as the membrane.

Figure S12 shows the applied voltage breakdown for the area normalized (current density) results. a) shows the iR-corrected polarization curves, b) shows the kinetic overpotential calculated from the Tafel analysis shown in the inset of Figure 1, c) shows the ohmic overpotential calculated from the HFR, and d) shows the mass-transport overpotential which is calculated as the balance of the overpotential. The onset of the severe mass-transport resistance can be seen near $1200 \mathrm{~mA} \mathrm{~cm}{ }^{-2}$ on the $0.011 \mathrm{mg}_{\text {Ir }} \mathrm{cm}^{-2}$ curve in d), and below $300 \mathrm{~mA} \mathrm{~cm}^{-2}$ for the lower loaded CCMs. 


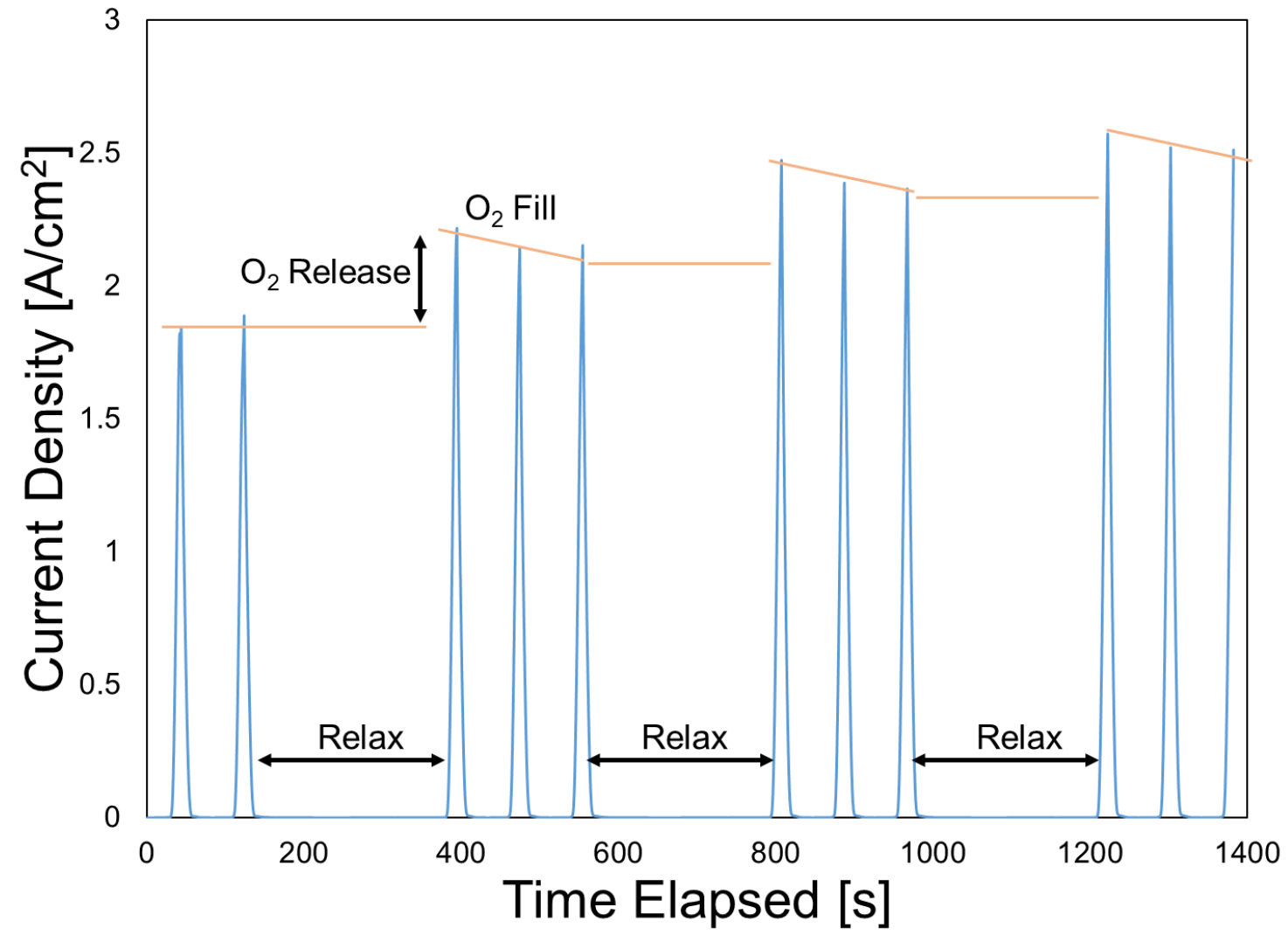

Figure S13. Variable relaxation time study to highlight the impact of oxygen bubbles on the anode. After each extended relaxation time the current density improved for the first cycle, and then degraded for subsequent cycles. Cell was held at $80{ }^{\circ} \mathrm{C}$, Ir loadings of $0.06 \mathrm{mg}_{\mathrm{Ir}} \mathrm{cm}^{-2}$. The cell was well activated and conditioned before the measurements were taken. 

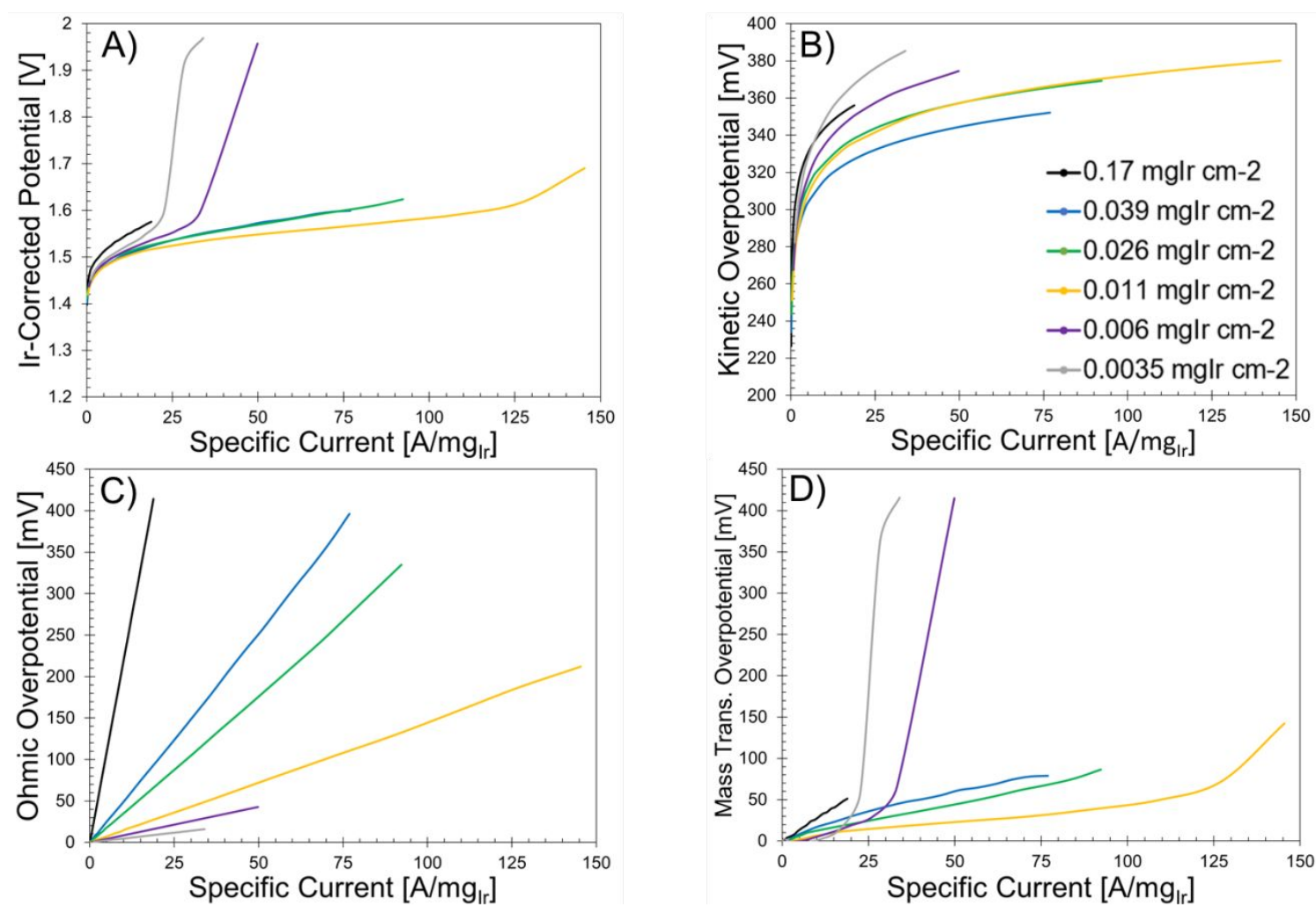

Figure S14. a) PEMWE iR-corrected polarization curves at different Ir loadings under constant ionomer/catalyst ratio of 0.116 ; b) kinetic overpotential, c) ohmic overpotential and d) mass transport overpotential extracted from data shown in Figure 6 and a). PEMWEs were tested under ambient pressure at $80{ }^{\circ} \mathrm{C}$. Cathode Pt loadings were kept at $0.2 \pm 0.02 \mathrm{mg} / \mathrm{cm}^{2}$ for all tests. Nafion ${ }^{\mathrm{TM}} 117$ was used as the membrane.

Normalizing the cell current by the mass of Ir metal reveals a different picture. The mass transport resistance for each of the CCMs is qualitatively similar up to a specific current of approximately $20 \mathrm{~A} \mathrm{mg}_{\text {Ir }}$ at which point the optimal loading of $0.011 \mathrm{mg}_{\mathrm{Ir}} \mathrm{cm}^{-2}$ maintains a low mas transport resistance.

At a specific current of over $125 \mathrm{~A} \mathrm{mg}_{\text {Ir }}$ the onset of the severe mass-transport resistance is seen. The lower loaded CCMs maintain even lower mass-transport resistance than the optimal CCM, but the onset of the severe limitation occurs at a much lower specific current. This indicates that the mass-transport limitation is located near the active sites themselves since the mass normalization is not able to fully resolve the trend. 
A)

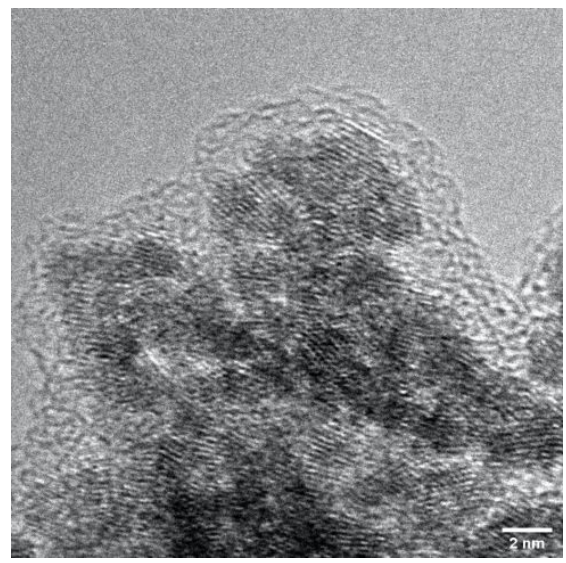

C)

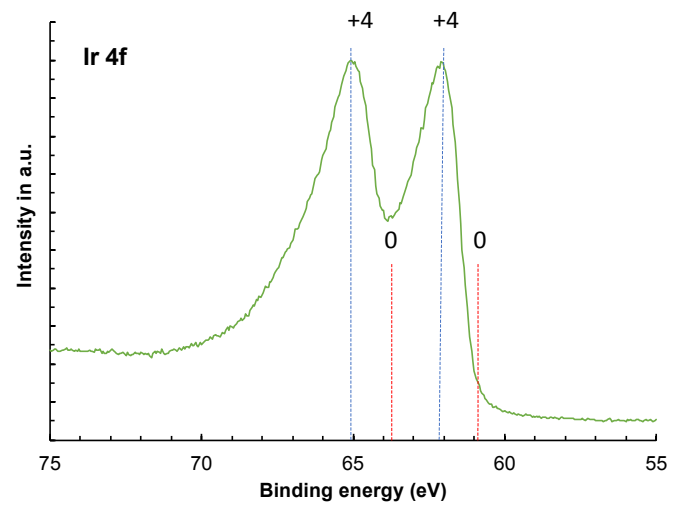

B)

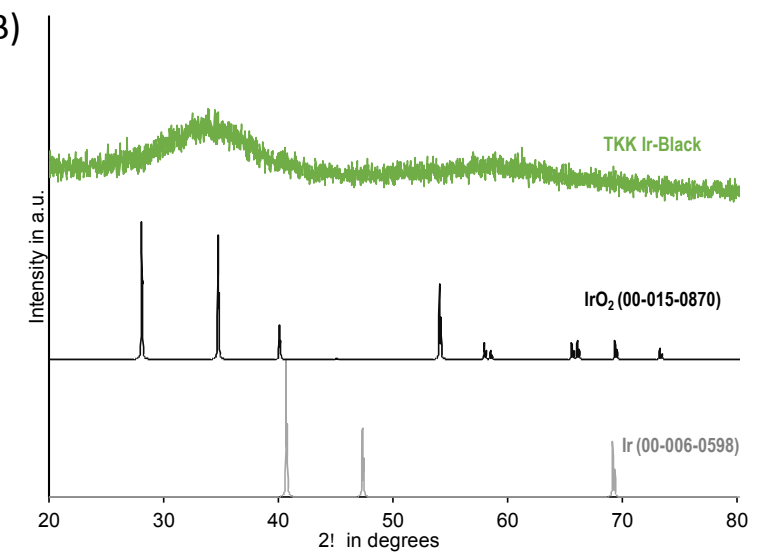

D)

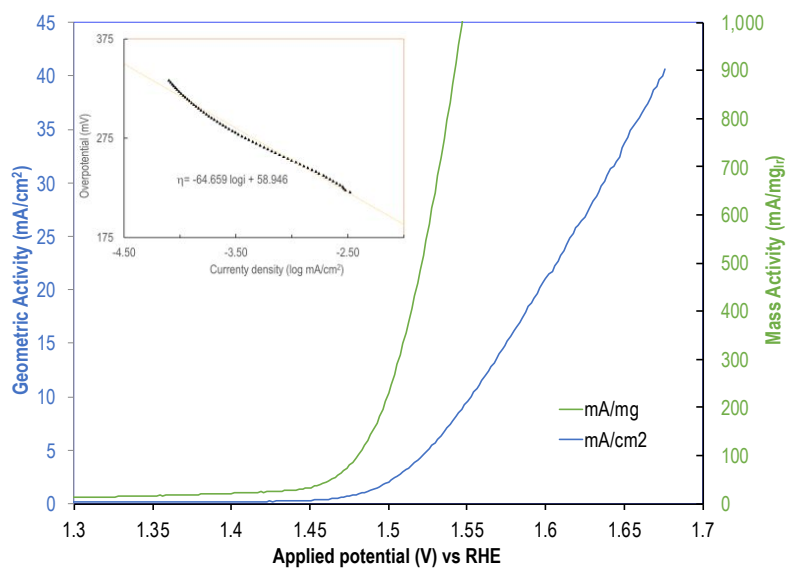

Figure S15. Initial characterization of TKK ELC-0110 SA=100 Ir catalyst used including a) Transmission electron microscope (TEM) image, b) X-ray diffraction (XRD), c) X-ray photoelectron spectroscopy (XPS), and d) rotating disk electrode (RDE) measurement in $0.1 \mathrm{M} \mathrm{HClO}_{4}$ at room temperature.

Figure S15 provides ex-situ characterization of the TKK ELC-0110 SA=100 catalyst used in this study. A) TEM imaging of the catalyst shows an amorphous structure near the catalyst surface which has been shown to enhance activity with a crystalline core. B) The XRD indicates the catalyst is predominantly $\mathrm{IrO}_{2}$ crystalline structure, with small crystalline domains and amorphous content. C) XPS of the Ir $4 \mathrm{f}$ shell shows spectra consistent with a +4 near surface Ir charge (reference lines for Ir metal shown). D) RDE testing shows an active OER catalyst by both geometric and mass normalized activity consistent with earlier reports ${ }^{3}$. We note that the geometric area normalized Tafel slope of $65 \mathrm{mV} \mathrm{dec}^{-1}$ (inset d)) which is higher than the $\sim 40 \mathrm{mV} \mathrm{dec}^{-1}$ recorded during MEA testing.

XRD plots are recorded in Bragg-Brentano (BB) optics geometry with a $\mathrm{Cu} \mathrm{K} \alpha$ source on a Rigaku SmartLab instrument. STEM micrographs were acquired using a $200 \mathrm{kV}$ FEI monochromated F20 UT Tecnai instrument. The TEM-EDX maps are collected on a FEI TitanX 60-300 microscope equipped with a Bruker windowless EDS detector with a solid angle of 0.7 steradians. XPS data are collected on a Kratos Axis Ultra DLD system at a takeoff angle of $0^{\circ}$ relative to the surface normal at room temperature. A monochromatic $\mathrm{Al} \mathrm{K \alpha}$ source $(\mathrm{h} v=1486.6 \mathrm{eV})$ is used to excite the core level 
electrons of the material. Pt 4f, Ir 4f, Ti 2p, C 1s, and O 1s core levels are collected, with a pass energy of $20 \mathrm{eV}$, step size of $0.05 \mathrm{eV}$, and 8 sweeps each to obtain a good signal to noise ratio. The survey spectra (Figure S1) are acquired with a pass energy of $160 \mathrm{eV}$, step size of $1 \mathrm{eV}$, and 3 sweeps. The measurements are performed at ultrahigh vacuum conditions $\left(7.5 \times 10^{-9}\right.$ Torr $)$.

Electrochemical measurement methods are adapted from earlier reports ${ }^{3}$. The gold disk working electrode is prepared by polishing with $0.05 \mu \mathrm{m}$ alumina slurry. The disk is rinsed and bath sonicated for $30 \mathrm{sec}$ to remove residual slurry and impurities. The disk is rinsed again and dried via a compressed nitrogen air gun. The glassware is cleaned by rinsing in ultra-pure water (18.2 M $\Omega$, Milli-Q), boiling for 1 $\mathrm{h}$, and rinsing again. Catalyst inks are coated as a thin film layer on a polished gold tip working electrode ( $5 \mathrm{~mm}$ diameter). To prepare the inks, catalyst powder $(3.5 \mathrm{mg})$ is combined with water $(7.6 \mathrm{~mL}), 2$ propanol (2.4 mL, VWR), and Nafion solution ( $40 \mu \mathrm{L}, 5 \mathrm{wt} \%$ in alcohol, Ion Power). The catalyst suspension was bath sonicated for $20 \mathrm{~min} .10 \mu \mathrm{L}$ of the well-dispersed catalyst ink was pipetted onto the gold disk surface of the working electrode as it rotates on an inverted RDE at $100 \mathrm{rpm}$ to distribute the ink evenly. The RDE speed is increased to $700 \mathrm{rpm}$ and allowed to dry for 15-20 min until it is completely dry. Electrochemical measurements are performed using a Pine Instrument RDE and SP-300 potentiostat from BioLogic Science Instruments. The activities are recorded in $0.1 \mathrm{M} \mathrm{HClO}_{4}$ electrolyte at $25{ }^{\circ} \mathrm{C}$. A gold wire and dynamic hydrogen electrode (DHE) are used as the counter and reference electrode, respectively. All potentials are adjusted to RHE. Once set up, the system is purged with ultra-high purity Ar for 15 min before the working electrode is placed in the electrolyte and electrochemical measurements are recorded. 50 preliminary cyclic voltammetry (CV) scans are recorded at a $500 \mathrm{mV} / \mathrm{s}$ between $0.025 \mathrm{~V}$ and $1.5 \mathrm{~V}$. OER activity CVs are then recorded at $20 \mathrm{mV} / \mathrm{s}$.

\section{References}

(1) Khandavalli, S.; Park, J. H.; Kariuki, N. N.; Zaccarine, S. F.; Pylypenko, S.; Myers, D. J.; Ulsh, M.; Mauger, S. A. Investigation of the Microstructure and Rheology of Iridium Oxide Catalyst Inks for Low-Temperature Polymer Electrolyte Membrane Water Electrolyzers. ACS Appl. Mater. Interfaces 2019. https://doi.org/10.1021/acsami.9b14415.

(2) Berlinger, S. A.; McCloskey, B. D.; Weber, A. Z. Inherent Acidity of Perfluorosulfonic Acid Ionomer Dispersions and Implications for Ink Aggregation. J. Phys. Chem. B 2018. https://doi.org/10.1021/acs.jpcb.8b06493.

(3) Alia, S. M.; Anderson, G. C. Iridium Oxygen Evolution Activity and Durability Baselines in Rotating Disk Electrode Half-Cells. J. Electrochem. Soc. 2019, 166 (4), F282-F294. https://doi.org/10.1149/2.0731904jes. 